the achieved reduction in exposure on the burden of chronic obstructive pulmonary disease (COPD) in construction workers. Method The intervention aimed at technical, organisational and psycho-social factors and was performed in four construction companies. Pre and post intervention respirable quartz exposure measurements were taken in these and four additional control companies. A mathematical simulation model was used to generate COPD prevalences (GOLD stage $\geq 1$ ) in response to silica exposure, population characteristics and Dutch trends in smoking behaviour for a population of 20-65 year old construction workers with lifetime silica exposure.

Results Pre-intervention exposure assessment demonstrated highest respirable quartz levels $\left(\mathrm{mg} / \mathrm{m}^{3}\right)$ for concrete drillers (GM: 0.20, GSD: 2.75), tuck pointers (GM: 0.18, GSD (2.18) and demolishers (GM: 0.12, GSD: 2.86), exceeding the Dutch occupational exposure limit (OEL) in 71, 92 and 97\% of cases, respectively. Preliminary simulations estimated COPD prevalence at 21,20 and $17 \%$ for these groups respectively, as compared to $14 \%$ when quartz exposure is reduced to the Dutch OEL and $8 \%$ with no exposure.

Conclusions For several job categories high exposure levels exceeding the Dutch OEL were observed. Reducing these levels to below the OEL would lead to a substantial reduction in the burden of disease. The post intervention exposure levels will become available early 2014. The effect on the burden of disease and economic impact will be assessed with an refined model incorporating population dynamics.

\section{CARPAL TUNNEL SYNDROME AND CARPAL TUNNEL SYNDROME-LIKE SYMPTOMS IN RELATION TO MECHANICAL EXPOSURES ASSESSED BY A JOB EXPOSURE MATRIX: A TRIPLE CASE-REFERENT STUDY}

${ }^{1}$ Sorosh Tabatabaeifar, ${ }^{2}$ Susanne Wulff Svendsen, ${ }^{3}$ Birger Johnsen, ${ }^{3}$ Anders Fuglsang-Frederiksen, ${ }^{1}$ Poul Frost. 'Danish Ramazzini Centre, Department of Occupational Medicine, Aarhus University Hospital, Aarhus, Denmark; ${ }^{2}$ Danish Ramazzini Centre, University Department of Occupational Medicine, Herning Regional Hospital, Herning, Denmark; ${ }^{3}$ Department of Neurophysiology, Aarhus University Hospital, Aarhus, Denmark

\subsection{6/oemed-2014-102362.264}

Objectives To evaluate relations between occupational mechanical exposures and (1) carpal tunnel syndrome verified by ENG (ENG+CTS) and (2) CTS-like symptoms with normal ENG (ENG-CTS).

Method We plan a triple-case referent study of 1000 ENG + CTS and 1000 ENG-CTS cases identified at a university department of clinical neurophysiology. For each case, two sex, age, and primary care centre matched controls will be sampled (risk set sampling). Both retrospectively and prospectively identified cases will be included with standardised clinical examination of the last-mentioned group. Conditional logistic regression analyses will be performed comparing the two case control sets, while unconditional logistic regression will be applied comparing ENG+CTS cases to ENG-CTS controls. Questionnaire information will be collected on job history, lifestyle, symptoms, and disability. Job titles will be linked to a job exposure matrix (JEM) based on measurements of hand-wrist movements (goniometer measurements) and expert ratings.

Results The main hypothesis is that exposure-response relations will be found for ENG+CTS, but not for ENG-CTS with respect to forceful work and awkward wrist postures, while repetitive work will show exposure-response relations in both groups. The Danish Working Environment Research Fund has granted financial support for a 3 -year $\mathrm{PhD}$ project starting January 2014.

Conclusions The study will take advantage of specific and well documented case diagnoses and independent exposure assessment. The results are expected to produce new insights into exposure-response relations between occupational mechanical exposures and risk of CTS.

\section{ARE INDOOR CONCENTRATIONS OF AIRBORNE MOULD SPORES IN NON-INDUSTRIAL ENVIRONMENTS SUFFICIENTLY HIGH TO CAUSE HYPERSENSITIVITY PNEUMONITIS?}

${ }^{1}$ Henrik A Kolstad, ${ }^{2}$ Simon Skov, ${ }^{3}$ Tine Birgitte Halsen Malling, ${ }^{4}$ Elisabeth Bendstrup, 1,5 Vivi Schlünssen. ${ }^{1}$ Department of Occupational Medicine, Danish Ramazzini Centre, Aarhus University Hospital, Aarhus, Denmark; ${ }^{2}$ Forest and Landscape, Faculty of Life Sciences, University of Copenhagen, Copenhagen, Denmark; ${ }^{3}$ Department of Occupational Medicine, Danish Ramazzini Centre, Aalborg University Hospital, Aalborg, Denmark; ${ }^{4}$ Department of Respiratory Medicine and Allergology, Aarhus University Hospital, Aarhus, Denmark; ${ }^{5}$ Department of Public Health, Section for Environment, Work, and Health, Danish Ramazzini Centre, Aarhus University, Aarhus, Denmark

\subsection{6/oemed-2014-102362.265}

Objectives Antigen exposure is the only diagnostic criteria specific for hypersensitivity pneumonitis (HP) compared with other interstitial lung diseases. Indoor mould exposure in non-industrial environments has been claimed to cause HP, but little is known about exposure levels. Our objective was to compare indoor concentrations of airborne mould spores for patients diagnosed with indoor HP with background levels and levels measured for patients diagnosed with farmers' lung and suberosis.

Method We included 8 patients diagnosed with HP based on characteristic clinical findings, signs of indoor mould growth at home or at their non-industrial work place, and increased levels of precipitating antibodies for moulds. We collected 110 air samples from all affected rooms, adjacent rooms, and outdoor.

Results The average total spore concentrations varied between 22000 and 36000 spores per $\mathrm{m}^{3}$, and the average viable concentrations between $35 \mathrm{CFU} / \mathrm{m}^{3}$ and $457 \mathrm{CFU} / \mathrm{m}^{3}$, with no clear association between spore concentration and affected rooms, adjacent rooms and outdoor.

Conclusions The observed levels did not exceed usual indoor and outdoor levels and were orders of magnitude below levels measured for patients diagnosed with farmers' lung and suberosis, and we question if indoor mould levels in non-industrial environments are sufficient to cause HP. Relying solely on signs of moulds or presence of precipitating antibodies when diagnosing HP may cause other interstitial lung diseases to be overseen and patients may take initiatives such as quitting the job or leaving their homes that will not alleviate their disease but have significant social consequences.

\section{PROFESSIONAL TRAJECTORY IS ASSOCIATED WITH MULTIPLE CARCINOGENIC EXPOSURES AT WORK AMONG MEN : DATA FROM A COHORT OF PATIENTS SUFFERING RESPIRATORY CANCER}

${ }^{1,2}$ Emilie Counil, ${ }^{3,4}$ Mélanie Bertin, ${ }^{3}$ The Giscop93 team. ${ }^{1}$ EHESP Rennes, Sorbonne Paris Cité, Paris, France; ${ }^{2}$ IRIS, UMR 8156-997, Bobigny, France; ${ }^{3}$ IRSET, UMR Inserm 1085, Rennes, France; ${ }^{4}$ Giscop93, Université Paris 13, Bobigny, France

10.1136/oemed-2014-102362.266 
Objectives Social position and social mobility are associated with cancer incidence and mortality, yet little is known about their association with mediating factors such as occupational exposures to carcinogens. Our aim was to assess the association between the type of professional trajectory and multiple occupational exposure profiles.

Method Data were extracted from the Giscop93 study ( $\mathrm{n}=1$ 009), which is a cohort of cancer patients with (mainly) respiratory tumours. Job histories were reconstructed through interview, then a multi-disciplinary expert group examined the probability of occupational exposure to a list of 54 potentially carcinogenic agents. The typology of professional trajectories was built based on employment stability, employment continuity, job qualification trend, and multiple skills through Multiple Correspondence Analysis followed by Ascending Hierarchical Classification. Association with multiple-exposure profiles was then assessed through multiple logistic regression.

Results Men and women differed in terms of predominant job category over the lifecourse $(68,2 \%$ of blue-collar-workers among men, 57,3\% of employees among women, $\mathrm{p}<0.0001)$. Professional trajectories were grouped in four classes as "stable qualified, employee" $(21,3 \%)$, "stable manual, independent bluecollar-worker" (24,4\%), "stable tiring, no gain in qualification" $(30,5 \%)$, and "very unstable, precarious" $(23,8 \%)$. Among men, the last two categories were associated with exposure to at least five different occupational carcinogens $\left(\mathrm{OR}_{\text {stable_tiring/stable_quali- }}\right.$ fied $\left.=2,0[1,3 ; 3,1], \mathrm{OR}_{\text {very_unstable/stable_qualified }}=2,6[1,6 ; 4,2]\right)$. No such association was found among women.

Conclusions The association found between the type of professional trajectory and multiple occupational exposures among men should be replicated among people not suffering cancer. Forthcoming analysis will investigate the gendered differences observed.

\section{MENTAL HEALTH INEQUALITIES BY INFORMAL EMPLOYMENT AND GENDER IN CENTRAL AMERICA}

\begin{abstract}
1,2María López-Ruiz, 1,2 José M Martínez, 1,2Fernando G Benavides, 2,3 Vanessa Puig-Barrachina, ${ }^{2,4}$ Marianela Rojas, ${ }^{2,5}$ Lucía Artazcoz. ${ }^{1}$ CIBER Epidemiología Y Salud Pública (CIBERESP), Barcelona, Spain; '2Universitat Pompeu Fabra, Barcelona, Spain; ${ }^{3}$ Vrije Universiteit, Brussels, Belgium; ${ }^{4}$ Universidad Nacional de Costa Rica, Heredia, Costa Rica; ${ }^{5}$ Agència de Salut Pública de Barcelona, Barcelona, Spain
\end{abstract}

\subsection{6/oemed-2014-102362.267}

Objectives To analyse the relationship between mental health and non-agricultural informal employment in Central America; and to examine whether patterns of association differ by gender Method Cross-sectional study of 8904 non-agricultural workers ( $48 \%$ women) based on the I Central American Survey of Working Conditions and Health of 2011. Employment profiles were created combining formal and informal characteristics: labour relationship (permanent employees, temporary employees, selfemployed, employers), social security coverage (yes, no), type of contract for employees (written, oral or no contract), company size for employers $(\leq 5,>5$ workers). Using logistic regression models, odds ratios (OR) of poor mental health (measured by GHQ12 questionnaire) and 95\% confidence intervals (95\% CI) were calculated by sex, adjusting for country and age, with employment profiles as independent variable. The reference group was permanent employees covered by social security with a written contract.

Results Around 37\% of women and 34\% of men reported poor mental health. In both sexes all profiles without social security coverage were associated with poor mental health except for permanent employees. Temporary employees covered by social security were associated with poor mental health if they have oral or no contract for women and men. Covered permanent employees with oral or no contract among women (OR: 1.70, 95\% CI:1.12-2.59) and covered self-employed among men (OR: 1.59, 95\% CI:1.03-2.46) were associated with poor mental health.

Conclusions In Central America health inequalities by employment profiles exist, principally for not being covered by social security, or having an oral or no contract for employees (main characteristics of informal employment). Few gender inequalities have been found.

\section{THE PARTICIPATION FOR SOLID WASTE MANAGEMENT APPROPRIATE MODEL OF UNDERGRADUATE IN SRINAKHARINWIROT UNIVERSITY, THAILAND}

Anong Hansakul. Srinakharinwirot University, Bangkok, Thailand

\subsection{6/oemed-2014-102362.268}

Objectives The objective was to study participatory for solid waste management of undergraduate students Faculty of Physical Education Srinakarinwirot University Ongkarak.

Method Collect the data by the questionnaire developed interview used as a tool to collect employed quantitative data. The content validity was improved and adjusted by the suggestion of the experts.

Results The result revealed that: the sample were women $75.5 \%$, age between $18-22$ years old, age average 19.75 years old (S. D. $=1.047, \operatorname{Min}=18, \operatorname{Max}=22$ ), the bin condition, or storage of waste provided by the University have various stains $81.5 \%$, minor is not covered or close incompletely $56.8 \%$ and $36.1 \%$ damaged, broken or leaking the trash. The storage of waste by University provide cause of nuisance $88.7 \%$; almost of foul smell $92.5 \%$, minor is nuisance form animals and insects such as flies, mice, etc., $36.6 \%$ and the other (the leachate outflow / waste overflow flooded / gruesome) $4.2 \%$. The knowledge level about solid waste management almost moderate level 53.5\%, minor was high level $45.5 \%$ and low level $1.1 \%$. The attitude level about solid waste management almost moderate $65.8 \%$, minor were low level $18.2 \%$, and high level $16.0 \%$. The practice behaviour level about solid waste management almost moderate $72.8 \%$, minor were high level 15.2\% and low level 12.0\%.

Conclusions Undergraduate students have the knowledge, but not implemented for separate the garbage, and the correct type of waste, uncooperative for separate the garbage because of that embarrassment and there is not enough knowledge about solid waste management. The University do not have the campaign seriously.

\section{PROSTATE CANCER RISK AMONG FRENCH FARMERS IN THE AGRICAN COHORT}

${ }^{1,2}$ Clémentine Lemarchand, 1,2Séverine Tual, 1,2 Noémie Levêque-Morlais, ${ }^{1,2}$ Stéphanie Perrier, ${ }^{1,3}$ Anne-Valérie Guizard, ${ }^{4}$ Michel Velten, ${ }^{5}$ Emma Rigaud, ${ }^{6,7}$ Isabelle Baldi, ${ }^{1,2}$ Pierre Lebailly. 'INSERM, UMR1086 Cancers Et Préventions, F-14000, Caen, France; ${ }^{2}$ University of Caen Basse-Normandie, F-14000, Caen, France; ${ }^{3}$ Calvados General Tumor Registry, Centre François Baclesse, F-14000, Caen, France; ${ }^{4}$ Bas-Rhin Cancer Registry, Faculty of Medicine, University of Strasbourg, F-67085, Strasbourg, France; ${ }^{5}$ Mutualité Sociale Agricole, Caisse Centrale, F-93547, Bagnolet, France; ${ }^{6}$ INSERM, ISPED, Centre INSERM U897- Epidemiologie-Biostatistique, F-33000, Bordeaux, France; 'University of Bordeaux, ISPED, Laboratoire Santé Travail Environnement, F-33000, Bordeaux, France

10.1136/oemed-2014-102362.269 